\title{
Hyperboloidal initial data for the vacuum Einstein equations with cosmological constant
}

\author{
János Kánnár† \\ Max-Planck-Institut für Gravitationsphysik, Albert-Einstein-Institut, Schlaatzweg 1, D-14473 \\ Potsdam, Germany \\ Received 7 May 1996, in final form 24 July 1996
}

\begin{abstract}
The existence of smooth hyperboloidal initial data sets for the vacuum Einstein equations with non-zero cosmological constant $\Lambda$ is studied. Supposing that the trace $\tilde{\chi}$ of the (physical) second fundamental form of the initial hypersurface is constant, there is a correspondence between the solutions of the vacuum constraints with and without cosmological constant, respectively. This enables us to extend the results proved by Andersson and Chruściel about the smoothness of the initial data with zero cosmological constant to the case $\Lambda \neq 0$.
\end{abstract}

PACS number: 0420E

\section{Introduction}

We shall consider vacuum spacetimes with cosmological constant and such spacelike hypersurfaces, called hyperboloidal hypersurfaces, which intersect conformal infinity (scri). We shall mainly work in the conformally compactified picture (see $[7,8]$ ) and study the solutions of the (conformal) constraint equations on hyperboloidal hypersurfaces. The case $\Lambda=0$ was first studied by Andersson et al [1]: they considered hypersurfaces where the physical second fundamental form was a pure trace, i.e. proportional to the metric. In order to get smooth initial data, the free data (in this case the conformal metric) have to satisfy certain boundary conditions on the conformal compactified initial hypersurface. The following three statements proved to be equivalent: the conformal initial data are smooth; the scri is shear-free; the electric part of the four-dimensional Weyl tensor is zero on the boundary of the initial hypersurface (because of the special situation the magnetic part is identically zero on the whole hypersurface). This result was generalized by Andersson and Chruściel [2] to such hypersurfaces where the only restriction on the physical second fundamental form is that its trace $\tilde{\chi}$ is a non-zero constant. They derived the boundary conditions which the free data, i.e. the conformal metric $h_{a b}$ and a symmetric trace-free covariant tensor field $A_{a b}$ (see section 3), have to satisfy in order to get smooth conformal initial data. In the present paper this result will be extended to the case of non-zero cosmological constant.

The vacuum spacetimes with non-zero cosmological constant differ in some sense significantly from the ones with $\Lambda=0$. For the latter, conformal infinity (if it is sufficiently smooth) can be represented as a null hypersurface of the conformally extended spacetime (see $[7,8]$ ), but (using signature $(-,+,+,+)$ ) conformal infinity is timelike or spacelike, when the cosmological constant is negative or positive, respectively. Despite this difference,

$\dagger$ On leave from KFKI Research Institute for Particle and Nuclear Physics, Budapest. 
the conditions which guarantee the smoothness of the initial data differ only slightly from the ones given by Andersson and Chruściel [2]. The main result of this paper, the smoothness conditions with $\Lambda \neq 0$, is contained in the following statement.

Theorem 1. Let $u_{0}$ be a conformal hyperboloidal initial data set (see definition 2 later) with smooth free data $\left(h_{a b}, A_{a b}\right)$ and with the gauge choices (2.7) and (3.6), to the vacuum conformal field equations with cosmological constant. Let $\Lambda$ satisfy the inequality $\Lambda<\frac{1}{3} \tilde{\chi}^{2}$ and let $\tilde{\chi}^{\prime}=\left(\tilde{\chi}^{2}-3 \Lambda\right)^{\frac{1}{2}}$. Suppose, furthermore, that the trace of the physical second fundamental form $\tilde{\chi}$ is a non-zero constant. In this case $u_{0}$ is smooth iff the conditions

(a)

$$
\tilde{\chi}^{\prime} \lambda_{A B}+\tilde{\chi} \chi_{A B}=0
$$

(b)

$$
\delta^{A} \delta^{B} \lambda_{A B}+R_{A B} \lambda^{A B}=0, \quad D_{1} \chi_{A B}-\frac{1}{2} h_{A B} h^{C D} D_{1} \chi_{C D}=0
$$

are satisfied on the boundary of the initial hypersurface $\partial M$ (see (2.7.3) and conditions (3.15) later).

Remark. Without loss of generality one can choose the tensor field $A_{a b}$ such that the components $A_{1 b}$ vanish on $\partial M$. Supposing this is true, the conditions (a) and (b) can be explicitly written in terms of the free data $\left(h_{a b}, A_{a b}\right)$ and they practically give restrictions on some of their derivatives normal to the boundary:

$\left(a^{\prime}\right)$

$$
\tilde{\chi}^{\prime} \lambda_{A B}+\tilde{\chi} A_{A B}=0
$$

$$
\delta^{A} \delta^{B} \lambda_{A B}+R_{A B} \lambda^{A B}=0, \quad D_{1} A_{A B}-\frac{1}{2} h_{A B} h^{C D} D_{1} A_{C D}=0 .
$$

Notation. The notation is essentially the same as, for example, in [1]. All the Latin tensor indices denote tetrad indices. $i, j, k, \ldots$ will denote four-dimensional, i.e. spacetime indices, and take the values $\{0,1,2,3\} . a, b, c, \ldots$ and $A, B, C \ldots$ are restricted to the hypersurface $\bar{M}$ and to its boundary $\partial M$, so they take the values $\{1,2,3\}$ and $\{2,3\}$, respectively. The Greek indices are tensor indices with respect to some coordinate system. $\nabla, D$ and $\delta$ mean the torsion free, metric covariant differential operators on the spacetime, on the initial hypersurface and on its boundary, respectively. The above equations contain the projections of the following quantities to $\partial M: \chi_{A B}$ and $\lambda_{A B}$ denote those of the second fundamental form of $\bar{M}$ and $\partial M$, respectively; $R_{A B}$ is that of the Ricci tensor, $h_{A B}$ and $A_{A B}$ of the free data on $\bar{M}$, i.e. of the conformal metric and the tensor field $A_{a b}$, respectively. The definition of the second fundamental form of $\partial M, \lambda_{A B}=e_{A}^{\alpha} e_{B}^{\beta} \nabla_{\alpha} e_{1 \beta}$, differs in sign from the one used in [1,2]. The physical quantities are distinguished with a tilde from their unphysical pairs and their indices are raised and lowered with the physical metric.

Conditions (b) and $\left(b^{\prime}\right)$ are the same as those for the vacuum case, but (a) and $\left(a^{\prime}\right)$, both of which express the vanishing of the shear of scri, differ slightly from the vacuum conditions (see [2]). These hold because of the following: supposing that the trace of the physical second fundamental form is constant, there is then a strong connection between the solutions of the constraints of the vacuum case with zero cosmological constant and those with $\Lambda \neq 0$. Given a solution to the former, then considering the cosmological constant $\Lambda$ as a parameter, we can generate a one-parameter set of solutions to the constraints with 
several values of $\Lambda$. The trace of the physical second fundamental form of the initial hypersurface $\tilde{\chi}$ varies also (in terms of $\Lambda$ ), such that the quantity $\tilde{\chi}^{\prime}=\left(\tilde{\chi}^{2}-3 \Lambda\right)^{\frac{1}{2}}$ remains fixed in a family of solutions characterized with the same $\Lambda=0$ vacuum solution.

When $\tilde{\chi}=0$ the situation differs from the cases considered in theorem 1 , because here one has to add an additional condition to the ones contained in the theorem to get smooth conformal initial data. One has to require that the trace-free part $L_{a b}$ of the conformal (unphysical) second fundamental form $\chi_{a b}$ be zero at the boundary of the initial hypersurface. This is exactly the asymptotic version of the condition which was imposed in [1] quoted at the beginning of the paragraph. Namely, there the considerations were restricted to such hyperboloidal initial hypersurfaces where $L_{a b}=0$ was satisfied over the whole hypersurface. Imposing $L_{a b}=0$ on $\partial M$ an analogue of the result of [1] can be proved. This is true independently of the value of the cosmological constant satisfying the inequality $\Lambda<\frac{1}{3} \tilde{\chi}^{2}$ and at the same time this includes also the extension of theorem 1 for the $\tilde{\chi}=0$ case. This result is formulated precisely in the following statement.

Theorem 2. Let $u_{0}$ be a conformal hyperboloidal initial data set with smooth free data $\left(h_{a b}, A_{a b}\right)$ and with the gauge choices (2.7) and (3.6) to the vacuum conformal field equations with cosmological constant. Let $\Lambda$ satisfy the inequality $\Lambda<\frac{1}{3} \tilde{\chi}^{2}$. Let us suppose that the trace-free part of the unphysical second fundamental form $L_{a b}$ vanishes on $\partial M$. In this case the following three statements are equivalent:

- $u_{0}$ is in $C^{\infty}(\bar{M})$;

- $\lambda_{A B}=0$ and $D_{1} \chi_{A B}-\frac{1}{2} h_{A B} h^{C D} D_{1} \chi_{C D}=0$ on $\partial M$

- the (four-dimensional) Weyl tensor vanishes on $\partial M$.

Once a smooth conformal initial data set $u_{0}$ is given, a unique smooth Cauchy evolution exists in some neighbourhood of the initial hypersurface, as shown by Friedrich [4]. He proved this for the $\Lambda=0$ case, but this is also true for non-zero cosmological constant because the evolution equations do not contain $\Lambda$, which appears only in the constraints. Considering the $\Lambda=0$ vacuum case the solution of the hyperboloidal initial-value problem in the past or in the future extends up to the conformal infinity whenever the hypersurface intersects past or future null infinity, respectively. However, scri is not a null hypersurface when the cosmological constant is non-zero. This means that, for example, in the case of a negative cosmological constant (anti-de Sitter type spacetimes) in order to get a solution up to the conformal boundary one has to solve an initial-boundary-value problem (see [6]).

\section{The initial-value problem}

In this section we define the initial-value problem precisely and introduce the formalism which we shall use. The next two definitions are both modifications of those given in [4]: they include the cosmological constant $\Lambda$, and they do not require smoothness of the data, only continuity. The very purpose of this paper is to find the additional conditions which one has to impose to obtain smooth initial data. The definition of the hyperboloidal initial data sets in terms of the physical quantities is as follows.

Definition 1. A triple $\left(M, \tilde{h}_{a b}, \tilde{\chi}_{a b}\right)$ is called a hyperboloidal initial data set to the vacuum Einstein equations with cosmological constant $\Lambda$ if $M$ is a manifold diffeomorphic to the open unit ball in $R^{3}, \tilde{h}_{a b}$ is a (positive definite) Riemannian metric and $\tilde{\chi}_{a b}$ is a symmetric covariant tensor field on $M$, such that

(a) the constraints induced by the field equations hold on $M$, i.e.

$$
\tilde{R}+\tilde{\chi}^{2}-\tilde{\chi}_{a b} \tilde{\chi}^{a b}-2 \Lambda=0, \quad \tilde{D}_{a}\left(\tilde{\chi}^{a}{ }_{b}-\tilde{h^{a}}{ }_{b} \tilde{\chi}\right)=0
$$


(b) there exists a conformal closure of this initial data set, i.e. $M$ may be diffeomorphically identified with the interior of a manifold $\bar{M}$ with boundary $\partial M$, where $\bar{M}$ is diffeomorphic to the closed unit ball in $R^{3}$ (whence $\partial M$ is diffeomorphic to the sphere $S^{2}$ ) and on $\bar{M}$ there exist functions $\Omega$ and $\Sigma$ such that

- $\Omega>0$ on $M, \Omega=0$ on $\partial M$,

- at least one of $\Sigma$ or $D_{a} \Omega$ is non-zero on $\partial M$,

- $h_{a b}=\Omega^{2} \tilde{h}_{a b}$ extends to an at least continuous Riemannian metric on $\bar{M}$,

- $\frac{1}{3} \Lambda-\Sigma^{2}+D^{a} \Omega D_{a} \Omega=0$ on $\partial M$,

- $\chi_{a b}=\Omega\left(\tilde{\chi}_{a b}+\Sigma \tilde{h}_{a b}\right)$ extends to an at least continuous tensor field on $\bar{M}$.

If $\Lambda \neq 0$, then the second condition of (b) follows from the fourth one. The above definition requires that the conformal metric $h_{a b}$ and the conformal second fundamental form $\chi_{a b}$ should extend at least continuously to the whole of $\bar{M}$. To get smooth initial data the fields $\tilde{\chi}_{a b}$ and $\tilde{h}_{a b}$ have also to satisfy certain 'fall-off' conditions. However, it seems more natural and convenient to treat the hyperboloidal initial-value problem in terms of the conformal quantities, working entirely on the conformally compactified manifold. In this way the question of the asymptotic behaviour of the several quantities near to infinity can be reduced to their local study at the points of $\partial M$. Instead of the Einstein equations we consider a first-order system initiated by Friedrich, the so-called conformal field equations (for their original form see [3] and for the equations with cosmological constant [6]), which contain the following as variables: the conformal factor $\Omega$, its gradient $\Sigma_{i}=\nabla_{i} \Omega, s=\frac{1}{4} \nabla_{i} \nabla^{i} \Omega$, an orthonormal tetrad field $\left\{e_{i}^{\alpha}\right\}$, the connection coefficients $\gamma_{j k}^{i}$, the quantity $s_{i j}=\frac{1}{2}\left(r_{i j}-\frac{1}{4} g_{i j} r\right)$ which is just half of the trace-free part of the unphysical Ricci tensor and the rescaled Weyl tensor $d_{i j k l}=C_{i j k l} / \Omega$. The vector $e_{0}^{\alpha}$ is the unit normal of the hypersurface $\bar{M}$ and the tetrad is parallel propagated along $e_{0}^{\alpha}$. Using the integral curves of $e_{0}^{\alpha}$ we can define a Gauss coordinate system $\{t, x, y, z\}$ in some neighbourhood of $\bar{M}$, where $\{t=0\}$ is the initial hypersurface $\bar{M}$.

Here we quote only the constraints, which are just the inner equations of the conformal field equations on the initial hypersurface $\bar{M}$ (see [5]):

$t_{a b}{ }^{c} e_{c}^{\mu}=0$,

$R_{a b}=-\chi \chi_{a b}+\chi_{a}^{c} \chi_{b c}+\Omega d_{a b}+s_{a b}+\left(\frac{1}{6} r+s_{00}\right) h_{a b}$,

$D_{b} \chi_{c a}-D_{c} \chi_{b a}=\Omega d_{a b c}+2 h_{a[b} s_{c]}$,

$D_{a} \Omega=\Sigma_{a}$

$D_{a} \Sigma_{b}=\Sigma \chi_{a b}-\Omega s_{a b}+s h_{a b}$

$D_{a} \Sigma=\Sigma^{c} \chi_{c a}-\Omega s_{a}$,

$D_{a} s=\Sigma s_{a}-\Sigma^{c} s_{c a}-\frac{1}{12} r \Sigma_{a}-\frac{1}{24} \Omega D_{a} r$,

$D_{a} s_{b}-D_{b} s_{a}=2 \chi_{[a}^{c} s_{b] c}+\Sigma^{c} d_{c a b}$,

$D_{a} s_{b c}-D_{b} s_{a c}=2 \chi_{c[a} s_{b]}+\Sigma d_{c a b}+\Sigma^{e} d_{e c a b}+\frac{1}{12} h_{c[a} D_{b]} r$,

$D^{c} d_{c a b}=2 d_{c[a} \chi_{b]}^{c}$,

$D^{c} d_{a c}=\chi^{c b} d_{c a b}$,

$6 \Omega s+\frac{1}{4} \Omega^{2} r+3 \Sigma^{2}-3 \Sigma_{a} \Sigma^{a}=\Lambda$,

$2 \Omega D^{a} D_{a} \Omega-3 D^{a} \Omega D_{a} \Omega+\frac{1}{2} \Omega^{2} R=-3 \Sigma^{2}-\frac{1}{2} \Omega^{2}\left(\chi^{2}-\chi_{a b} \chi^{a b}\right)+2 \Omega \Sigma \chi+\Lambda$.

(The last equation is just a consequence of the others, but we have included it in the system because it will be often used in the later calculations.) The above system contains the following unknowns:

$$
u_{0}=\left(e_{a}^{\alpha}, \gamma_{b c}^{a}, \chi_{a b}, \Omega, \Sigma, \Sigma_{a}, s, s_{a}, s_{a b}, d_{a b}, d_{a b c}\right) .
$$


$\chi_{a b}=e_{a}^{\alpha} e_{b}^{\beta} \nabla_{\alpha} e_{0 \beta}$ is the (unphysical) second fundamental form of $\bar{M}$. Together with this quantity the following projections with respect to $e_{0}^{\alpha}$ are used: $\Sigma=\Sigma_{0}, s_{a}=s_{0 a}, d_{a b}=$ $d_{a 0 b 0}, d_{a b c}=d_{a 0 b c}$ (the last two are essentially the electric and the magnetic part of the rescaled Weyl tensor with respect to the normal of $\bar{M}) . t_{a}{ }^{c}{ }_{b}$ is the torsion tensor of the covariant differential operator $D$, and it vanishes identically because $D$ is the Levi-Civita differential operator of the metric $h_{a b}$.

The conformal hyperboloidal initial data sets are described in the following manner.

Definition 2. A pair $\left(\bar{M}, u_{0}\right)$ is called a conformal hyperboloidal initial data set to the vacuum Einstein equations with cosmological constant $\Lambda$ if $\bar{M}$ is a manifold diffeomorphic to the closed unit ball in $R^{3}$ with boundary $\partial M$ and if the components of $u_{0}$ in (2.4) are a collection of fields on $\bar{M}$ such that

(a) $u_{0}$ satisfies the constraints induced by the conformal equations on $M=\bar{M} \backslash \partial M$;

(b) $\Omega>0$ on $M=\bar{M} \backslash \partial M$ and $\Omega=0$ on $\partial M$, but at least one of $\Sigma$ or $D_{a} \Omega$ is non-zero on $\partial M$ (in the $\Lambda \neq 0$ case the latest follows from the former condition).

The smoothness of the conformal initial data on $\bar{M}$ guarantees the existence of their unique local time evolution [4]. We will give conditions (boundary conditions on $\partial M$ ) on some components of $u_{0}$, which are necessary and sufficient to the smoothness of the initial data set $u_{0}$ on the whole of $\bar{M}$.

A solution of the above system can be generated as follows: let us suppose that we have a solution to the physical vacuum constraints with cosmological constant, i.e. the pair $\left(\tilde{h}_{a b}, \tilde{\chi}_{a b}\right)$ is a solution of equations (2.1). After some calculations one can show that their conformal transformed pair $\left(h_{a b}, \chi_{a b}\right)$, with some appropriate functions $\Omega$ and $\Sigma$, satisfy the equations

$R+\chi^{2}-\chi_{a b} \chi^{a b}-4 s_{a}{ }^{a}-\frac{1}{2} r=0, \quad D_{b}\left(\chi_{a}^{b}-\delta^{b}{ }_{a} \chi\right)-2 s_{a}=0$.

These are just the contractions of $(2.3 .2 a)$ and $(2.3 .2 b)$, respectively, supposing that the emerging quantities $s_{a b}$ and $s_{a}$ were calculated from the constraints (2.3.4a) together with (2.3.8) and from $(2.3 .4 b)$, respectively. Others of the above constraint equations can also be considered as the definitions of the further, yet unknown, components of $u_{0}$, namely of $\left(\Sigma_{a}, s, d_{a b}, d_{a b c}\right)$. The conformal constraint equations (2.3) are not all independent, but they satisfy some relations, of which all but one are algebraic (these are almost the same as those for the case $\Lambda=0$ in [4]). Because of these connections the remaining equations of system (2.3) also hold.

Let us suppose now that a hyperboloidal initial data set as in definition 1 is given. This means that the conformal quantities $\left(h_{a b}, \chi_{a b}, \Omega, \Sigma\right)$ and from them some components of $u_{0}$, namely $\left(e_{a}^{\alpha}, \gamma_{b c}^{a}, \chi_{a b}, \Omega, \Sigma\right)$, are known directly. The remaining quantities, i.e. $\left(\Sigma_{a}, s, s_{a}, s_{a b}, d_{a b}, d_{a b c}\right)$, as was mentioned before, can be calculated using some of the constraint equations just as their definitions. But before doing this explicitly we can make some simplifications to system (2.3).

So far we have not fixed the conformal gauge (i.e. the freedom that the triple $\left(\overline{\mathcal{M}}, \theta^{2} g_{i j}, \theta \Omega\right)$, for all positive $\theta$, determines the same physical spacetime $\left.\left(\mathcal{M}, \tilde{g}_{i j}\right)\right)$, so the Ricci scalar $r$ of the four-dimensional unphysical metric was a free function in the above equations. Now we choose it to be

$$
r=2 R
$$

where $R$ denotes the Ricci scalar of the three-dimensional conformal metric on the $\{t=$ constant $\}$ hypersurfaces, i.e. during the study of the constraints $R$ is the Ricci scalar on $\bar{M}$. The trace of the physical second fundamental form $\tilde{\chi}$ is supposed to be a constant. 
Using its conformal transformation rule the trace of the conformal second fundamental form becomes

$$
\chi=\frac{3}{\Omega}\left(\frac{\tilde{\chi}}{3}+\Sigma\right) .
$$

But $\Sigma$ can be fixed freely on the initial hypersurface. It is useful to choose

$$
\Sigma=-\frac{1}{3} \tilde{\chi} \quad \text { on } \bar{M} \text {. }
$$

(On $\partial M$ one has no other possible choice to extend $\chi$ up to the boundary.) In this case the conformal second fundamental form is trace-free, i.e.

$$
\chi=0 \quad \text { on } \bar{M},
$$

which means that in this gauge $\chi_{a b}$ and its trace-free part $L_{a b}$ can be interchanged.

Taking the above simplifications into account we can calculate the set $\left(\Sigma_{a}, s, s_{a}, s_{a b}, d_{a b}, d_{a b c}\right)$ as functions of $\left(h_{a b}, \chi_{a b}, \Omega, \Sigma\right)$ :

$$
\begin{aligned}
& \Sigma_{a}=D_{a} \Omega, \\
& s=\frac{1}{3} D^{a} D_{a} \Omega-\frac{1}{12} \Omega \chi_{a b} \chi^{a b}, \\
& s_{c}=\frac{1}{2} D^{a} \chi_{a c}, \\
& s_{a b}=\frac{1}{\Omega}\left\{-\left(D_{a} D_{b} \Omega-\frac{1}{3} h_{a b} D^{c} D_{c} \Omega\right)-\frac{\tilde{\chi}}{3} \chi_{a b}\right\}-\frac{1}{12} h_{a b} \chi_{c d} \chi^{c d}, \\
& d_{a b}=\frac{1}{\Omega}\left\{\left(R_{a b}-\frac{1}{3} h_{a b} R\right)-\chi_{a}^{c} \chi_{b c}+\frac{1}{4} h_{a b} \chi_{c d} \chi^{c d}-s_{a b}\right\}, \\
& d_{a b c}=\frac{1}{\Omega}\left\{D_{b} \chi_{c a}-D_{c} \chi_{b a}+\frac{1}{2} h_{a c} D^{d} \chi_{d b}-\frac{1}{2} h_{a b} D^{d} \chi_{d c}\right\} .
\end{aligned}
$$

Supposing that the unphysical metric $h_{a b}$ is smooth (this holds a priori in the following, because the process of solving the (physical) constraints starts with a smooth unphysical metric), in order to get a $C^{\infty}$ conformal initial data set $u_{0}$, we have to study only the behaviour of the quantities $\left(\Omega, \chi_{a b}, s_{a b}, d_{a b}, d_{a b c}\right)$.

\section{On the solutions of the constraints}

In their paper, Andersson and Chruściel [2] have studied the solutions of the vacuum constraints in the cases where the trace of the second fundamental form $\tilde{\chi}$ was taken to be a non-zero constant. Their results are easily applicable for non-vanishing cosmological constant as well.

Let us denote by $\tilde{L}_{a b}$ the trace-free part of the (physical) second fundamental form, i.e. $\tilde{\chi}_{a b}=\tilde{L}_{a b}+\frac{1}{3} \tilde{h}_{a b} \tilde{\chi}$. Because $\tilde{\chi}$ is supposed to be a constant, the (physical) vacuum constraint equations (2.1) take the form

$$
\tilde{D}_{b} \tilde{L}^{b}{ }_{a}=0, \quad \tilde{R}+\frac{2}{3} \tilde{\chi}^{2}-\tilde{L}_{a b} \tilde{L}^{a b}-2 \Lambda=0 .
$$

Let us suppose, furthermore, that the cosmological constant satisfies $\Lambda \leqslant \frac{1}{3} \tilde{\chi}^{2}$. This holds for all the anti-de Sitter type problems and also for some de Sitter like cases when $\tilde{\chi} \neq 0$. Then equations (3.1) can be written as

$$
\tilde{D}_{b} \tilde{L}^{b}{ }_{a}=0, \quad \tilde{R}+\frac{2}{3}\left(\tilde{\chi}^{2}-3 \Lambda\right)-\tilde{L}_{a b} \tilde{L}^{a b}=0,
$$

which look like the ordinary vacuum constraints with second fundamental form

$$
\tilde{\chi}_{a b}^{\prime}=\tilde{L}_{a b}+\frac{1}{3} \tilde{h}_{a b} \tilde{\chi}^{\prime}, \quad \text { where } \tilde{\chi}^{\prime}=\left(\tilde{\chi}^{2}-3 \Lambda\right)^{\frac{1}{2}} .
$$


Considering this transformation from the opposite point of view, we can say that whenever we have a solution $\left(\tilde{h}_{a b}, \tilde{\chi}_{a b}=\tilde{L}_{a b}+\frac{1}{3} \tilde{h}_{a b} \tilde{\chi}^{\prime}\right)$ to the physical vacuum constraints with a constant $\tilde{\chi}^{\prime}$, then $\left(\tilde{h}_{a b}, \tilde{\chi}_{a b}=\tilde{L}_{a b}+\frac{1}{3} \tilde{h}_{a b} \tilde{\chi}\right)$, where $\tilde{\chi}=\left(\tilde{\chi}^{\prime 2}+3 \Lambda\right)^{\frac{1}{2}}$, is a solution with a cosmological constant $\Lambda$, supposing that $\Lambda \geqslant-\frac{1}{3} \tilde{\chi}^{\prime 2}$. Because of its special behaviour, the case $\tilde{\chi}=0$ will be separately studied at the end of this section. For now we assume that $\tilde{\chi} \neq 0$.

Using the Lichnerowitz-Choquet-Bruhat-York method, one starts with a set $\left(\bar{M}, h_{a b}, A_{a b}, \rho\right)$, where

- $\bar{M}$ is a manifold diffeomorphic to the closed unit ball in $R^{3}$ with boundary $\partial M$,

- $h_{a b}$ is a (positive definite) $C^{\infty}$ Riemannian metric on $\bar{M}$,

- $A_{a b}$ is an arbitrary symmetric, traceless, $C^{\infty}$ tensor field on $\bar{M}$,

- $\rho$ is a $C^{\infty}$ defining function of $\partial M: \rho>0$ on $M ; \rho=0, D_{a} \rho \neq 0$ on $\partial M$.

The function $\rho$ can be chosen so that the Ricci scalar of the metric $h_{a b}^{*}=\left(1 / \rho^{2}\right) h_{a b}$ satisfies

$$
R\left(h^{*}\right)=-6+R_{3} \rho^{3}, \quad \text { where } R_{3} \in C^{\infty}(\bar{M}) .
$$

After possible rescaling of $\rho$ and $h_{a b}$ by some smooth positive factor $\theta$ (i.e. $\rho \rightarrow \theta \rho, h_{a b} \rightarrow$ $\theta^{2} h_{a b}$ ), we can get, in addition to the above, that

$$
D^{a} \rho D_{a} \rho=1 \quad \text { in some neighbourhood of } \partial M .
$$

This means that $x=\rho$ can be chosen in some neighbourhood of $\partial M$ as the Gauss coordinate which is normal to the boundary and the tetrad vectors in this region can be chosen to be parallel propagated along $e_{1}^{\alpha}=D^{\alpha} x$. Using $h_{a b}$ and $\rho$ with these features, we get, for the trace $\lambda$ of the second fundamental form $\lambda_{a b}$ of the $\{x=$ constant $\}$ surfaces, that

$$
\lambda=0, \quad \frac{\partial \lambda}{\partial x}=-\frac{1}{4} R \quad \text { on } \partial M .
$$

As we have seen in the second section a solution to the conformal constraints with $\Lambda \neq 0$ can be generated easily, by making a conformal transformation and using the formulae (2.8), from a solution of the physical vacuum constraint (3.1). Furthermore, as was explained earlier, the solutions to the physical vacuum constraints with cosmological constant can be generated from the solutions of the vacuum constraints without cosmological constant. So first we treat these physical vacuum constraints, which we start by solving the equation

$$
D_{a}\left[\frac{1}{x^{3}}\left(D^{a} X^{b}+D^{b} X^{a}-\frac{2}{3} D_{c} X^{c} h^{a b}\right)+\frac{1}{x^{2}} B^{a b}\right]=0,
$$

for $X^{a}$, supposing that $B^{a b}=A^{a b}$. Then, defining

$$
X^{a b}=\frac{1}{x^{3}}\left(D^{a} X^{b}+D^{b} X^{a}-\frac{2}{3} D_{c} X^{c} h^{a b}\right)+\frac{1}{x^{2}} B^{a b},
$$

the scalar equation for $\Omega$,

$$
\Omega D^{a} D_{a} \Omega-\frac{3}{2} D^{a} \Omega D_{a} \Omega+\frac{1}{4} \Omega^{2}\left(R-\Omega^{4} X^{a b} X_{a b}\right)+\frac{3}{2} \varepsilon^{2}=0,
$$

taking $\varepsilon=\frac{1}{3} \tilde{\chi}^{\prime}$ should be solved. Considering

$$
L^{a b}=\Omega^{2} X^{a b},
$$

the physical quantities

$$
\tilde{h}_{a b}=\frac{1}{\Omega^{2}} h_{a b}, \quad \tilde{\chi}_{a b}=\frac{1}{\Omega} L_{a b}+\frac{1}{\Omega^{2}} h_{a b} \frac{\tilde{\chi}}{3},
$$

where

$$
\tilde{\chi}=\left(\tilde{\chi}^{\prime 2}+3 \Lambda\right)^{\frac{1}{2}}
$$

solve the vacuum constraints (3.1) with cosmological constant $\Lambda$. 
In [2] Andersson and Chruściel solved (proved the existence of and characterized the solutions to) the above problem in the case $\varepsilon=1$ for arbitrary smooth $B^{a b}$. Let us suppose that the cosmological constant satisfies the inequality

$$
\Lambda<\frac{1}{3} \tilde{\chi}^{2} .
$$

This is a technical condition: by now excluding the equality we assure that the constant $\tilde{\chi}^{\prime}=\left(\tilde{\chi}^{2}-3 \Lambda\right)^{\frac{1}{2}}$ is non-zero. This ensures the applicability of the results of [2]. With $B^{a b}=\left(\frac{1}{3} \tilde{\chi}^{\prime}\right)^{2} A^{a b}$ and $\varepsilon=1$ let us denote the solutions of the equations (3.8)-(3.10) with $\bar{X}^{a b}, \bar{\Omega}$ and $\bar{L}^{a b}$, respectively. Then it is easy to see that the solutions in our case, i.e. with $B^{a b}=A^{a b}$ and $\varepsilon=\frac{1}{3} \tilde{\chi}^{\prime}$, are $X^{a b}=\left(\frac{1}{3} \tilde{\chi}^{\prime}\right)^{-2} \bar{X}^{a b}, \Omega=\frac{1}{3} \tilde{\chi}^{\prime} \bar{\Omega}$ and $L^{a b}=\bar{L}^{a b}$. In general, these solutions are not smooth at $\partial M$, but $\Omega \in C^{3}(\bar{M})$ and $L^{a b} \in C^{1}(\bar{M})$. Near $\partial M$ they cannot be written simply as power series of the coordinate $x$, but as a series also containing logarithmic terms, powers of $\log (x)$. Including the (3.6) gauge condition the conformal factor $\Omega$ near $\partial M$ takes the form

$\Omega=\frac{1}{3} \tilde{\chi}^{\prime}\left\{x-\frac{1}{12} L_{A B} L^{A B} x^{3}-\frac{1}{8}\left[R_{A B} \lambda^{A B}+\delta^{A} \delta^{B} \lambda_{A B}-L^{A B} D_{1} L_{A B}\right] x^{4} \log (x)+\right.$ h.o.t. $\}$.

Let us suppose (also taking into account the gauge choices (2.7) and (3.6)) that

$$
\tilde{\chi}^{\prime} \lambda_{A B}+\tilde{\chi} L_{A B}=0 \quad \text { on } \partial M,
$$

which means that scri is shear-free (because one can get (3.15.1) through the decomposition of the condition $\nabla_{i} \nabla_{j} \Omega-\frac{1}{4} g_{i j} \nabla_{k} \nabla^{k} \Omega=0$ on $\partial M$ ). In this case, where neither $\tilde{\chi}$ nor $\tilde{\chi}^{\prime}$ can be zero (i.e. $L_{A B}$ is proportional to $\lambda_{A B}$ ), the smoothness of the data $\Omega$ and $L_{a b}$ is equivalent to the conditions

$$
\begin{aligned}
& \delta^{A} \delta^{B} \lambda_{A B}+R_{A B} \lambda^{A B}=0 \quad \text { on } \partial M ; \\
& D_{1} L_{A B}-\frac{1}{2} h_{A B} h^{C D} D_{1} L_{C D}=0 \quad \text { on } \partial M .
\end{aligned}
$$

(The second condition we have written with covariant derivative, instead of the coordinate derivative which was used in [2], because this form is more suitable to the following calculations.) Choosing free data such that $A_{1 b}=0$ does not restrict the generality. Writing conditions (3.15) in terms of $A_{a b}$ instead of the trace-free part of the second fundamental form $L_{a b}$ they look like

$$
\begin{aligned}
& \tilde{\chi}^{\prime} \lambda_{A B}+\tilde{\chi} A_{A B}=0 \quad \text { on } \partial M ; \\
& \delta^{A} \delta^{B} \lambda_{A B}+R_{A B} \lambda^{A B}=0 \quad \text { on } \partial M ; \\
& D_{1} A_{A B}-\frac{1}{2} h_{A B} h^{C D} D_{1} A_{C D}=0 \quad \text { on } \partial M .
\end{aligned}
$$

Now we are in the position to prove the theorems formalized in the introduction. First we show that the (3.15) conditions (or the equivalent (3.16) ones) also guarantee the smoothness of the whole conformal initial data set $u_{0}$. From (2.8.4) we can see that, under the above conditions, $s_{a b}$ is smooth iff $D_{a} D_{b} \Omega-\frac{1}{3} h_{a b} D^{c} D_{c} \Omega=-\frac{1}{3} \tilde{\chi} L_{a b}$ (because of (2.7) $L_{a b}$ and $\chi_{a b}$ are exchangeable). After its $(2+1)$ decomposition at $\partial M$, using the expression (3.14) of $\Omega$, we get the following three requirements:

$$
L_{11}=0, \quad L_{1 A}=0, \quad \tilde{\chi}^{\prime} \lambda_{A B}+\tilde{\chi} L_{A B}=0 .
$$

The first two are just consequences of the (2.3.4b) constraint equation and the (2.7.2) choice of $\Sigma$ (which yields $\chi_{a b} \equiv L_{a b}$ ). The last one is our first condition (3.15.1), so the smoothness 
of $s_{a b}$ really follows from (3.15). Using L'Hospital's rule one can determine the concrete form of $s_{a b}$ :

$s_{11}=-\frac{1}{12} R+\frac{1}{4} L_{A B} L^{A B}-\left(\tilde{\chi} / \tilde{\chi}^{\prime}\right) D_{1} L_{11}$,

$s_{1 A}=-\left(\tilde{\chi} / \tilde{\chi}^{\prime}\right) D_{1} L_{1 A}$

$s_{A B}=R_{A B}-\frac{1}{3} h_{A B} R-\frac{1}{2} h_{A B} \lambda^{C D} \lambda_{C D}-\frac{1}{4} h_{A B} L_{C D} L^{C D}-\left(\tilde{\chi} / \tilde{\chi}^{\prime}\right) D_{1} L_{A B}$.

From formulae (2.8.5) and (2.8.6), one can see that $d_{a b}$ and $d_{a b c}$ are smooth iff $C_{a b}=0$ and $C_{a b c}=0$ at $\partial M$, respectively, i.e. the electric and the magnetic parts of the Weyl tensor vanish on the boundary. Substituting the (2.8.3) form of $s_{a}$ into constraint (2.3.4b) and using L'Hospital's rule we can find that

$$
D_{1} \chi_{a 1}=\frac{1}{2} D^{b} \chi_{a b}
$$

on the boundary. From this result, by $(2+1)$ decomposition, we have the formulae

$$
D_{1} L_{11}=-\lambda_{A B} L^{A B}, \quad D_{1} L_{A 1}=\delta^{B} L_{B A},
$$

which are used in the later calculations. These expressions together with the (3.18) values of $s_{A B}$ give us, from definitions (2.8.5) and (2.8.6),

$$
C_{11}=0, \quad C_{1 B}=0, \quad C_{A B}=\left(\tilde{\chi} / \tilde{\chi}^{\prime}\right)\left(D_{1} L_{A B}-\frac{1}{2} h_{A B} h^{C D} D_{1} L_{C D}\right),
$$

for the electric part and

$$
\begin{aligned}
& C_{A B C}=0, \\
& C_{A B 1}=-\left(D_{1} L_{A B}-\frac{1}{2} h_{A B} h^{C D} D_{1} L_{C D}\right)-\left(L_{A C} \lambda^{C}{ }_{B}-\frac{1}{2} h_{A B} L_{C D} \lambda^{C D}\right), \\
& C_{1 B C}=L_{B D} \lambda^{D}{ }_{C}-L_{C D} \lambda^{D}{ }_{B}, \quad C_{11 C}=0,
\end{aligned}
$$

for the magnetic part of the Weyl tensor. Taking into account 'the zero shear' and the 'smoothness' conditions, i.e. (3.15), and using the identity $\lambda_{A C} \lambda^{C}{ }_{B}=\frac{1}{2} h_{A B} \lambda_{C D} \lambda^{C D}$, one can see that all the components of the Weyl tensor vanish on $\partial M$. This means that, similarly to the case of vanishing cosmological constant, conditions (3.15) are really enough to guarantee the smoothness of a conformal hyperboloidal initial data set $u_{0}$. The converse is also true: a smooth set $u_{0}$ satisfies conditions (3.15). Indeed, the 'zero shear' follows from the smoothness of $s_{a b}$ and the other two conditions are implied by the smoothness of $\Omega$ and $L_{a b}$.

The Weyl tensor on $\partial M$ vanishes whenever the conformal initial data $u_{0}$ are in $C^{\infty}(\bar{M})$ (or equivalently conditions (3.15) are satisfied). But the converse does not hold: a zero Weyl tensor on $\partial M$ does not imply the smoothness of $u_{0}$. Indeed, considering (2.8.5), from $C_{a b}=0$ it follows that $s_{a b}$ is at least in $C^{1}(\bar{M})$. Because $\Omega$ is in $C^{3}(\bar{M})$, the 'zero shear' condition (3.15.1) follows from (2.8.4) and the differentiability of $s_{a b}$. Formulae (3.20) and (3.21) for the components of the Weyl tensor hold even in this case. All this means that the vanishing of the Weyl tensor on $\partial M$ guarantees the vanishing of the shear of scri, but neither the smoothness of $L_{a b}$ nor that of $\Omega$, because (3.15.2) is not guaranteed to hold. (In the special case considered in [1], where the conformal second fundamental form $L_{a b}$ is identically zero on $\bar{M}$ and $\Lambda$ is zero, the vanishing of the Weyl tensor on $\partial M$ is equivalent to the smoothness of the conformal initial data $u_{0}$. Here (3.15.2) is satisfied trivially.)

Finally, we shall consider the special case when the trace of the conformal second fundamental form vanishes, i.e. $\tilde{\chi}=0$ (this requires that the cosmological constant should be non-zero, because $\tilde{\chi}^{\prime}$ is not allowed to vanish). In this case the 'zero shear' condition (3.15.1) takes the form

$$
\lambda_{A B}=0 \quad \text { on } \partial M \text {. }
$$


Conditions (3.15.2) and (3.15.3) guarantee the smoothness of $\Omega$ and $L_{a b}$ only if $L_{A B}$ is proportional to $\lambda_{A B}$ (see [2]). This means that we have to impose an additional condition, namely that

$$
L_{A B}=0 \quad \text { on } \partial M .
$$

Because of the choice (2.7.2) of $\Sigma$, i.e. $\chi=0$ on $\bar{M}$ and $\chi_{1 a}=0$ on $\partial M$ (from (2.3.4b)), this is equivalent to the condition

$$
\chi_{a b}=0 \quad \text { on } \partial M \text {. }
$$

The first of the 'smoothness' conditions (3.15.2) is simply a consequence of (3.22), i.e. the conformal factor $\Omega$ is automatically smooth when (3.22) and (3.23) (or equivalently (3.22) and (3.24)) hold. In order to make $L_{a b}$ also smooth, we need only impose condition (3.15.3). The same formulae as in the general, $\tilde{\chi} \neq 0$, case remain true (of course with the simplification $\tilde{\chi}=0$ ), so under conditions (3.22), (3.23) and (3.15.3) the initial data $u_{0}$ are smooth. However, in contrast to the $\tilde{\chi} \neq 0$ case, the converse does not hold: with smooth $u_{0}$ condition (3.23) is not necessarily satisfied.

Let us restrict ourselves to the case of such initial hypersurfaces, where $\chi_{a b}=0$ (i.e. $\left.L_{a b}=0\right)$ on the boundary. This is the asymptotic version of the condition which was imposed in [1]. In this case, from (3.22) we find that (3.15.1) and (3.15.2) are satisfied. It is easy to see from the above that for this class of initial hypersurfaces the following three conditions, independently of the value of the cosmological constant (satisfying only the inequality $\Lambda<\frac{1}{3} \tilde{\chi}^{2}$ ), are really equivalent:

- $u_{0}$ is in $C^{\infty}(\bar{M})$,

- $\lambda_{A B}=0$ and $D_{1} L_{A B}-\frac{1}{2} h_{A B} h^{C D} D_{1} L_{C D}=0$ on $\partial M$,

- the Weyl tensor vanishes on $\partial M$.

\section{Acknowledgments}

I would like to thank $\mathrm{H}$ Friedrich for suggesting the problem and for helpful discussions, Rachel Capon for checking the language of the paper and the referees for their remarks. The author of this work is a member of the research groups OTKA F014196 and OTKA T016246.

\section{References}

[1] Andersson L, Chruściel P T and Friedrich H 1992 On the regularity of solutions to the Yamabe equation and the existence of smooth hyperboloidal initial data for Einstein's field equations Commun. Math. Phys. 149 $587-612$

[2] Andersson L and Chruściel P T 1994 On hyperboloidal Cauchy data for vacuum Einstein equations and obstructions to smoothness of scri Commun. Math. Phys. 161 533-68

[3] Friedrich H 1981 The asymptotic characteristic initial value problem for Einstein's vacuum field equations as an initial value problem for a first-order quasi-linear symmetric hyperbolic system Proc. R. Soc. A 378 401-21

[4] Friedrich H 1983 Cauchy problems for the conformal vacuum field equations in general relativity Commun. Math. Phys. 91 445-72

[5] Friedrich H 1988 On static and radiative spacetimes Commun. Math. Phys. 119 51-73

[6] Friedrich H 1995 Einstein equations and conformal structure: existence of anti-de Sitter-type spacetimes J. Geom. Phys. 17 125-84

[7] Penrose R 1963 Asymptotic properties of fields and spacetimes Phys. Rev. Lett. 10 66-8

[8] Penrose R 1965 Zero rest mass fields including gravitation Proc. R. Soc. A 284 159-203 